\title{
Bose-Einstein Condensation of Long-Lifetime Polaritons in Thermal Equilibrium
}

\author{
Yongbao Sun, ${ }^{1, *}$ Patrick Wen, ${ }^{1}$ Yoseob Yoon, ${ }^{1}$ Gangqiang Liu, ${ }^{2}$ Mark Steger, ${ }^{2}$ Loren N. Pfeiffer, ${ }^{3}$ \\ Ken West, ${ }^{3}$ David W. Snoke, ${ }^{2, \dagger}$ and Keith A. Nelson ${ }^{1}$ \\ ${ }^{1}$ Department of Chemistry and Center for Excitonics, Massachusetts Institute of Technology, \\ 77 Massachusetts Avenue, Cambridge, Massachusetts 02139, USA \\ ${ }^{2}$ Department of Physics, University of Pittsburgh, 3941 O'Hara Street, Pittsburgh, Pennsylvania 15218, USA \\ ${ }^{3}$ Department of Electrical Engineering, Princeton University, Princeton, New Jersey 08544, USA
}

(Received 3 October 2016; published 5 January 2017)

\begin{abstract}
The experimental realization of Bose-Einstein condensation (BEC) with atoms and quasiparticles has triggered wide exploration of macroscopic quantum effects. Microcavity polaritons are of particular interest because quantum phenomena such as BEC and superfluidity can be observed at elevated temperatures. However, polariton lifetimes are typically too short to permit thermal equilibration. This has led to debate about whether polariton condensation is intrinsically a nonequilibrium effect. Here we report the first unambiguous observation of BEC of optically trapped polaritons in thermal equilibrium in a high- $Q$ microcavity, evidenced by equilibrium Bose-Einstein distributions over broad ranges of polariton densities and bath temperatures. With thermal equilibrium established, we verify that polariton condensation is a phase transition with a well-defined density-temperature phase diagram. The measured phase boundary agrees well with the predictions of basic quantum gas theory.
\end{abstract}

DOI: 10.1103/PhysRevLett.118.016602

The realization of exciton-polariton condensation in semiconductor microcavities from liquid-helium temperature [1,2] all the way up to room temperature [3-5] presents great opportunities both for fundamental studies of many-body physics and for all-optical devices on the technology side. Polaritons in a semiconductor microcavity are admixtures of the confined light modes of the cavity and excitonic transitions, typically those of excitons in semiconductor quantum wells placed at the antinodes of the cavity. Quantum effects such as condensation [1-5], superfluidity [6], and quantized vortices [7-11] have been reported. The dual light-matter nature permits flexible control of polaritons and their condensates, facilitating applications in quantum simulation. It is also straightforward to measure the spectral functions, $A(k, \omega)$, of polaritons, which can provide insights into the dynamics of many-body interactions in polariton systems. For cold atoms, the equilibrium occupation numbers can be measured [12], but the spectral function is not readily accessible. Observations of non-Hermitian physics [13] and phase frustration [14] have shown that polaritons are an important complement to atomic condensates.

However, in most previous experiments, the lifetime of the polaritons in microcavities has been 30 ps or less [15] due to leakage of the microcavity. Thus, although there have been claims to partial thermalization of polaritons [16,17], no previous work has unambiguously shown a condensation in thermal equilibrium, leading to the common description of polariton condensates as "nonequilibrium condensates" [18-20]. The theory of nonequilibrium condensation is still an active field [21-24]. Although polariton experiments and theory have shown that a great number of canonical features of condensation persist in nonequilibrium, e.g., superfluid behavior [22,23], some aspects may not [25,26], and debates persist over whether polariton condensates can be called Bose-Einstein condensates [27-29], in part related to the question of whether polariton condensation is intrinsically a nonequilibrium effect. It is thus of fundamental importance to investigate whether polariton condensates can reach thermal equilibrium. Of course, strictly speaking, BoseEinstein condensation (BEC) cannot occur in an ideal infinite $2 \mathrm{D}$ system, but it has been shown [30,31] that a 2D Bose gas in a large but finite trap has the same threshold behavior as a 3D Bose gas in a finite trap of the same type. We can thus talk of an equilibrium BEC in $2 \mathrm{D}$ and $3 \mathrm{D}$ finite trapped systems using the same language.

Trapping polaritons in a high- $Q$ microcavity.-The main challenge in reaching full thermalization in polariton systems is to achieve a very long polariton lifetime, longer than their thermalization time. The thermalization time for the polariton gas was estimated to be at least 40 ps for polaritons that are mostly excitonlike [16], and can be even longer for more-photonlike polaritons which are less interactive. However, most samples used in previous experiments have polariton lifetimes on the order of a few picoseconds. This suggests that an improvement of the cavity $Q$ by at least an order of magnitude is needed, which is not trivial for GaAs fabrication technology. We have succeeded at this by growing a GaAs-based high- $Q$ microcavity structure by molecular beam epitaxy. The main change from samples used in previous experiments [2] was to double the number of the quarter-wavelength layers in the distributed Bragg reflectors that make up the mirrors of the cavity; the detailed recipe and the difficulties involved 
in fabricating a long lifetime sample are described in the Supplemental Material [32]. The new microcavity structure has a $Q$ of $\sim 320000$ and a cavity photon lifetime of $\sim 135 \mathrm{ps}$. This corresponds to a polariton lifetime of 270 ps at resonance, which has been confirmed by the long-range (millimeter-scale) propagation of polaritons created through either resonant [48] or nonresonant [49] optical excitation.

Because of the light effective mass and inefficient scattering with phonons, polaritons can propagate over long distances, up to millimeters when they are mostly photonic $[48,49]$. In order to guide them toward equilibrium with a specified location and geometry, we made a spatial trap. We created an annular optical trap to localize polaritons under nonresonant excitation. This method has been used previously in several experiments to confine polaritons [50-54]. The excitation pattern on the microcavity is an annulus with a diameter of $19 \mu \mathrm{m}$, as shown in Fig. 1(a). In Fig. 1(b), we show the normalized light intensity plot for the $x=0$ slice of the ring pattern in Fig. 1(a). The light intensity in the center is nearly negligible; as discussed above, it gives rise to a nearly flat potential for the polaritons in the center of the ring, in which the variation in energy is much less than $k_{B} T$. We also plotted the intensity of the PL emitted by lower polaritons as a function of both the PL energy and the sample position for the case of moderate pump power below the condensation threshold in Fig. 1(c). The white dashed line indicates the emission energies at very low pump powers; the slope of this line arises from the wedge of the cavity thickness, which causes a gradient in the cavity photon energy. At the pump region, there is a blue shift of the polariton energy due to their interactions with each other as well as from repulsive interactions between polaritons and excitons and free carriers. As seen in

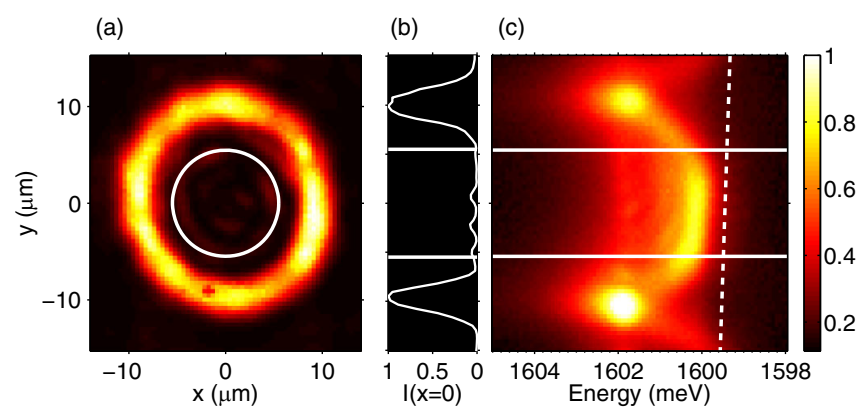

FIG. 1. (a) Reflection of the excitation beam from the sample surface. The white circle indicates the region of the sample that is observed in photoluminescence (PL) imaging measurements after spatial filtering. (b) Normalized excitation light intensity along the $x=0$ line through the center of the excitation ring pattern shown in (a). (c) Spectrally resolved PL along $x=0$. The PL within the solid white lines is collected and imaged onto the spectrometer CCD in the far-field geometry for the polariton distribution measurements. The dashed white line indicates the photon energy gradient deduced from the low-density spectrum.
Fig. 1(c), the barrier is not constant around the ring, varying by about $1 \mathrm{meV}$ from one side to the other due to inhomogeneity in the pump intensity. The barrier is slightly wider than the laser profile, because excitons propagate up to $10 \mu \mathrm{m}$. The potential landscape is nearly flat in the region from $-11 \mu \mathrm{m}$ to $11 \mu \mathrm{m}$ indicated by the white circle in Fig. 1(a) and the horizontal lines in Figs. 1(b) and 1(c). PL was collected from only this region for determination of the polariton energy distribution, as discussed below. The nearly flat potential profile corresponds to a constant density of states in 2D. Additionally, a nearly homogeneous distribution was established in the field of view, as evidenced by little change in the energy-resolved emission intensities in Fig. 1(c) (see Fig. S6 in Ref. [52] for a direct measurement of spatial profiles under similar conditions).

Polaritons are generated in the pump region and stream away in all directions. However, only polaritons that propagate into the center of the trap can meet and interact, leading to the accumulation of the densities high enough for condensation. A near-field image of the sample was projected onto a spatial filter at a reconstructed real-space plane of the sample surface to select only the PL from inside the trap [within the white circle in Fig. 1(a)], and a far-field image of the PL that passed through the spatial filter was projected onto an imaging spectrometer, giving the intensity of the PL, $I$, as a function of both the in-plane wave vector component, $k_{\|}$, and the corresponding energy, $E\left(k_{\|}\right)$. The dispersion $E\left(k_{\|}\right)$is given in the Supplemental Material [32]. Finally, $I\left[E\left(k_{\|}\right)\right]$was converted into the number of polaritons, $N\left[E\left(k_{\|}\right)\right]$[hereafter simply $\left.N(E)\right]$, by the use of one single efficiency factor throughout the experiments. Detailed information about how to determine the efficiency factor can be found in Ref. [52]. Crucially, the same efficiency factor was used for all the distributions so that the absolute occupation numbers of different distributions could be compared.

Varying the polariton gas from nonequilibrium to equilibrium.-To see the effect of interactions on thermalization, $N(E)$ was measured at two different cavity detunings, $\delta=-5 \mathrm{meV}$ and $\delta=0 \mathrm{meV}$, for a series of pump powers. The detuning $\delta$ is the energy difference between the cavity resonance and exciton energy at $k_{\|}=0$. Changing the detuning changes the underlying excitonic fraction of the polaritons, which governs the strength of their interactions. Positive values of detunings indicate polaritons are more excitonlike, while negative values of detunings give polaritons which are mostly photonlike. Here $\delta=0 \mathrm{meV}$ and $\delta=-5 \mathrm{meV}$ correspond to excitonic fractions of $50 \%$ and $30 \%$, respectively. This indicates that the polaritons with $\delta=-5 \mathrm{meV}$ have interactions which are weaker by a factor of 3 than those at $\delta=0 \mathrm{meV}$, and less well thermalized is expected.

The measured distributions $N(E)$ at both detunings and various pump powers are shown in Fig. 2. The pump powers are reported in terms of the threshold power, $P_{\mathrm{BE}}$, 

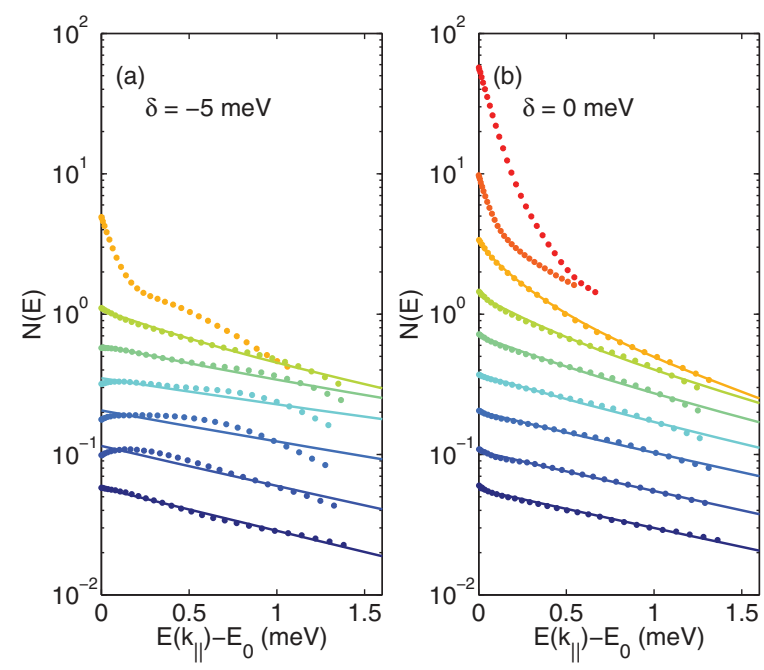

FIG. 2. Energy distributions of polaritons in the center of the trap at (a) $\delta=-5 \mathrm{meV}$ (weak interactions, nonequilibrium) and (b) $\delta=0 \mathrm{meV}$ (strong interactions, nonequilibrium) at a bath temperature of $T_{\text {bath }}=12.5 \mathrm{~K}$ at different pump powers (see Supplemental Material for values [32]). The solid curves are best fits to the equilibrium Bose-Einstein distribution in Eq. (1). The fitted values of $T$ and $\mu$ are shown in Fig. 3. The power values from low to high are $0.12,0.24,0.45,0.71,0.93,1.07,1.10,1.12$, and 1.14 times of the threshold values $P_{\mathrm{BE}}$, which are $382 \mathrm{~mW}$ and $443 \mathrm{~mW}$ for detunings $\delta=5 \mathrm{meV}$ and $\delta=0 \mathrm{meV}$, respectively.

defined below. The sample was immersed in a helium bath that was kept at a temperature $T_{\text {bath }}=12.5 \mathrm{~K}$ for both detuning positions. The measured values of $N(E)$ were fit to a Bose-Einstein distribution, given by

$$
N_{\mathrm{BE}}(E)=\frac{1}{e^{(E-\mu) / k_{B} T}-1},
$$

where $T$ and $\mu$ are the temperature and chemical potential of the polaritons, respectively, and $k_{B}$ is the Boltzmann constant. The ground state $\left(k_{\|}=0\right)$ of the lower polariton shifts to higher energy as the density increases, due to the repulsive interpolariton interactions. We defined the ground-state energy in each case as $E=0$, so that $\mu=0$ corresponds to the condition for Bose-Einstein condensation. The best fits of the data to $N_{\mathrm{BE}}(E)$ were determined using $T$ and $\mu$ as free parameters in nonlinear least-squares regressions, and are shown as solid curves in Fig. 2.

When the polariton states are negatively detuned and have very weak interactions, the fits to the Bose-Einstein distribution are poor. As seen in Fig. 2(a), for the case of $\delta=-5 \mathrm{meV}$, at low density the distribution has a reasonable fit to a Maxwell-Boltzmann distribution (which corresponds to a single exponential, i.e., a straight line on a semilog plot), but as the polariton density is increased, the distribution is no longer fully thermal. The hump at $E=0.5 \mathrm{meV}$ is a manifestation of the bottleneck effect, as was also observed in Ref. [17]. As the density is increased further, a peak occurs which is condensatelike, but the rest of the distribution does not fit the Bose-Einstein functional form in Eq. (1), indicating that the polaritons are not in thermal equilibrium. This behavior is similar to that seen in many other experiments with short-lifetime polaritons, e.g., Refs. [1,17], and is consistent with a nonequilibrium polariton condensate. The nonequilibrium distribution has been reproduced by numerical solution to the quantum Boltzmann equation [55]. Despite the long cavity lifetime, the photonlike polaritons with weak interactions do not reach thermal equilibrium.

In contrast, $N(E)$ at $\delta=0$ is well described by $N_{\mathrm{BE}}(E)$ for all pump powers up to $P=1.1 P_{\mathrm{BE}}$. At pump powers well below $P_{\mathrm{BE}}, N(E)$ is well described by a single exponential function, i.e., a Maxwell-Boltzmann distribution. Between $P=0.9 P_{\mathrm{BE}}$ and $P=1.1 P_{\mathrm{BE}}$, an upturn in the distribution at $E=0 \mathrm{meV}$ is observed, indicating that $N(E)$ deviates from Maxwell-Boltzmann statistics and must be described by a Bose-Einstein distribution with the reduced chemical potential $\left|\mu / k_{B} T\right|<1$. The fit values of $T$ and $\mu$ used in Fig. 2(b) are shown in Fig. 3 as the blue symbols. As seen in this figure, when the density is increased, $T$ decreases from around $20 \mathrm{~K}$ to a lowest value of $13.9 \pm 0.2 \mathrm{~K}$ and $\mu / k_{B} T$ smoothly goes from $-2.93 \pm$ 0.16 to $-0.28 \pm 0.01$. For pump powers greater than $1.1 P_{\mathrm{BE}}$, a condensate in the ground state appears. In the weakly interacting limit, the condensate peak should be delta-function-like, which is broadened in the presence of finite-size fluctuations [56]. The high-energy tail of the top two curves has the same absolute value, indicating that the population in the excited states saturates when there is a condensate, consistent with a Bose-Einstein condensation phase transition for bosons in thermal equilibrium.

The upturn in the shape of $N(E)$ in the low-energy states unambiguously distinguishes $N(E)$ as a Bose-Einstein distribution rather than a Maxwell-Boltzmann distribution. Previous reports using short-lifetime samples [16,17] showed fits of $N(E)$ but did not show this behavior;
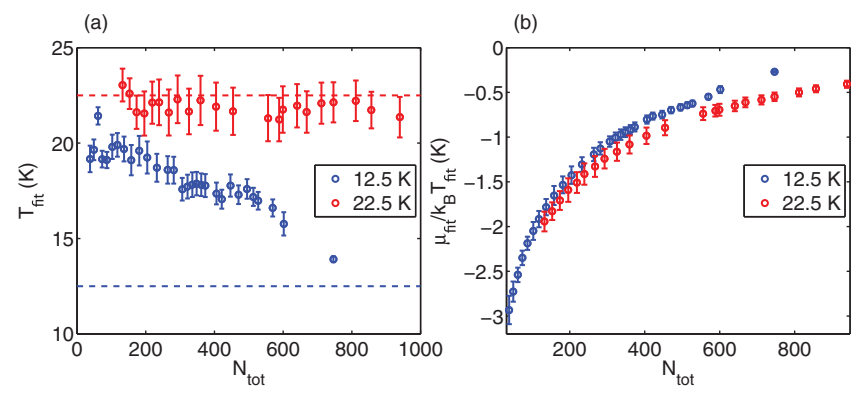

FIG. 3. (a) Effective temperatures of polaritons for bath temperatures $T=12.5 \mathrm{~K}$ (blue points) and $T=22.5 \mathrm{~K}$ (red points) at different pump powers, extracted by fitting the energy distributions [shown in Fig. 2(b) for the 12.5-K case] to the equilibrium Bose-Einstein model. The dashed lines indicate the helium bath temperatures. (b) Reduced chemical potential $\alpha=\mu / k_{B} T$ for bath temperatures $T=12.5 \mathrm{~K}$ (blue points) and $T=22.5 \mathrm{~K}$ (red points) at different pump powers. 
although a condensate peak appeared in some cases, there was not a clear density-dependent evolution from a thermal Maxwell-Boltzmann distribution to a degenerate Bose-Einstein condensed distribution. Furthermore, shortlifetime samples thermalized only when the microcavity was positively detuned [16] and the polariton characteristics were mostly excitonlike, so that the motion of the polaritons was severely restricted (see the Supplemental Material [32] for a detailed discussion). In contrast, the long-lifetime polaritons seen here at zero detuning follow Bose-Einstein statistics throughout the phase transition and propagate to fill the trap in spatial equilibrium.

We emphasize that not only the curvature of the fits in Fig. 2 but also the absolute vertical scale of the fits is constrained by the value of $\mu$. We do not have a free parameter to change the overall intensity scaling factor for each curve. The data points give the absolute occupation numbers as indicated by the vertical scale in addition to the relative occupation numbers at different pump powers. When the value of $\mu$ in the Bose-Einstein distribution is increased toward zero, this increases the absolute value of $N_{\mathrm{BE}}(E)$. Thus, the fits are tightly constrained by the requirement that we fit not only the shape of the distribution but also the relative heights of all the curves with only two parameters, $T$ and $\mu$. This constraint is reflected in the very small relative uncertainties in the fit values of $\mu$ shown in Fig. 3(b).

Phase diagram of polariton Bose condensation.-The bath temperature was also varied in the range of $10.0-25.0 \mathrm{~K}$. Good thermalization has been achieved across this range. In Fig. 3, we plot the fitted values of $T$ and $\mu / k_{B} T$ for different pump powers. As can be seen, when the bath temperature is low, the fit values of $T$ at low densities are much higher than $T_{\text {bath }}$ and at higher densities they settle to temperatures slightly above $T_{\text {bath }}$, while for a bath temperature of $T=22.5 \mathrm{~K}$, the fitted temperatures stay pinned to the bath temperature, within the uncertainty. The chemical potential increases smoothly toward zero in each case as the density is increased.

Now that we have a well-defined temperature ranging over which thermal equilibrium is established, it is straightforward to determine the phase diagram of polariton Bose-Einstein condensation. To determine the phase diagram, i.e., we want to plot the total number of polaritons $N$ as a function of the fit value of $T$ at the threshold, to check the expected scaling law that the particle separation should be of the order of the de Broglie wavelength at the threshold:

$$
r_{s} \sim n^{-1 / 2} \sim \lambda_{T} \sim \sqrt{\frac{\hbar^{2}}{m k_{B} T}} .
$$

We choose the threshold as the onset of Bose amplification, i.e., $N\left(k_{\|}=0\right)=1$.

Based on this methodology, $N_{\mathrm{BE}}$ were determined for a series of $T_{\text {bath }}$ values ranging from 10.0 to $25.0 \mathrm{~K}$. The fit
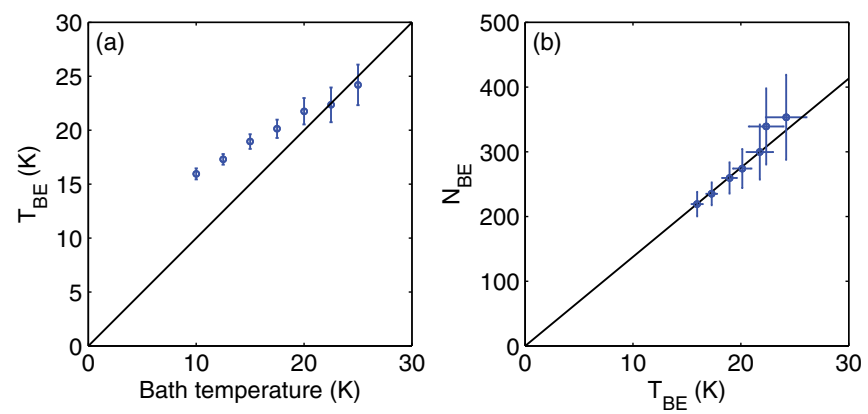

FIG. 4. (a) The critical temperature as a function of lattice temperature for $\delta=0 \mathrm{meV}$. The solid black line indicates $T_{\mathrm{BE}}=T_{\text {bath }}$. (b) Phase diagram of the transition to a degenerate Bose gas. The solid black line shows the best fit of a linear relation $N_{\mathrm{BE}} \propto T_{\mathrm{BE}}$.

values of $T$ at the onset of Bose-Einstein statistics are plotted in Fig. 4(a), showing the general trend of $T_{\mathrm{BE}}$ slightly higher than $T_{\text {bath }}$, as discussed earlier in the text. The relative deviation is highest at low bath temperature, when the heat capacity of the sample is lowest, allowing the local sample temperature to rise more due to the laser heating.

The phase diagram of Bose-Einstein transition, i.e., the relation of $N_{\mathrm{BE}}$ to $T_{\mathrm{BE}}$, is shown in Fig. 4(b). The black line is the best fit of a linear proportionality. Within the uncertainty, the data are consistent with a linear increase in threshold $T$ with $N_{\mathrm{BE}}$, consistent with the expected phase boundary of a weakly interacting boson gas in two dimensions implied by the relation (2). This line can be viewed as a phase boundary: above the line, the gas is quantum degenerate, and below it, the gas is classical.

It has been a long-standing assumption that the Bose condensation effects seen in polariton systems are a direct result of the quantum nature of the system when $r_{s} \sim \lambda_{T}$, but up to now it has not been possible to directly test this. By using high-quality microcavities with lifetimes over an order of magnitude longer than those of previous samples, polaritons within a two-dimensional flat optical trap are seen to unambiguously show thermal Bose-Einstein statistics. This clearly distinguishes polariton condensation from the conventional lasing effect in semiconductor materials.

Now that we have samples in which true equilibrium can be established, more new experiments are possible to test theoretical predictions of interacting Bose gases which have been elusive in cold atom experiments. Additionally, studies can be conducted of the excitation spectrum of the interacting Bose gas, and of the crossover from 2D to $1 \mathrm{D}$ equilibrium which can be controlled by spatial shaping of the excitation light to make tailored potential energy landscapes. Characteristics of the nonequilibrium state can also be studied systematically by varying the cavity detuning to control the polariton interaction strength and the excitation profile to tailor the potential landscape. Dynamical relaxation into the equilibrium state can also 
be studied by using pulsed rather than continuous-wave excitation followed by time-resolved measurements, as well as the coherence properties as the system passes through the Berezinskii-Kosterlitz-Thouless transition. The results are also encouraging for applications in quantum simulations of condensed matter systems that exploit equilibrium BEC properties [32].

This work was supported as part of the Center for Excitonics, an Energy Frontier Research Center funded by the U.S. Department of Energy, Office of Science, Office of Basic Energy Sciences under Award No. DE-SC0001088, by the National Science Foundation under Grants No. PHY-1205762 and No. DMR-1104383, by the Gordon and Betty Moore Foundation through the EPiQS initiative Grant No. GBMF4420, and by the National Science Foundation MRSEC Grant DMR-1420541.

*ybsun@mit.edu

†snoke@pitt.edu

[1] J. Kasprzak et al., Bose-Einstein condensation of exciton polaritons, Nature (London) 443, 409 (2006).

[2] R. Balili, V. Hartwell, D. Snoke, L. Pfeiffer, and K. West, Bose-Einstein condensation of microcavity polaritons in a trap, Science 316, 1007 (2007).

[3] S. Christopoulos et al., Room-Temperature Polariton Lasing in Semiconductor Microcavities, Phys. Rev. Lett. 98, 126405 (2007).

[4] S. Kena-Cohen and S. R. Forrest, Room-temperature polariton lasing in an organic single-crystal microcavity, Nat. Photonics 4, 371 (2010).

[5] J. D. Plumhof, T. Stoferle, L. Mai, U. Scherf, and R. F. Mahrt, Room-temperature Bose-Einstein condensation of cavity exciton-polaritons in a polymer, Nat. Mater. 13, 247 (2014).

[6] A. Amo, J. Lefrere, S. Pigeon, C. Adrados, C. Cuiti, I. Carusotto, R. Houdre, E. Giabino, and A. Bramati, Superfluidity of polaritons in semiconductor microcavities, Nat. Phys. 5, 805 (2009).

[7] K. G. Lagoudakis, M. Wouters, M. Richard, A. Baas, I. Carusotto, R. Andre, L. S. Dang, and B. Deveaud-Pledran, Quantized vortices in an exciton-polariton condensate, Nat. Phys. 4, 706 (2008).

[8] K. G. Lagoudakis, T. Ostatnický, A. V. Kavokin, Y. G. Rubo, R. André, and B. Deveaud-Plédran, Observation of half-quantum vortices in an exciton-polariton condensate, Science 326, 974 (2009).

[9] D. Sanvitto et al., Persistent currents and quantized vortices in a polariton superfluid, Nat. Phys. 6, 527 (2010).

[10] G. Nardin, G. Grosso, Y. Leger, B. Pietka, F. Morier-Genoud, and B. Deveaud-Pledran, Hydrodynamic nucleation of quantized vortex pairs in a polariton quantum fluid, Nat. Phys. 7, 635 (2011).

[11] G. Tosi, G. Christmann, N. G. Berloff, P. Tsotsis, T. Gao, Z. Hatzopoulos, P. G. Savvidis, and J. J. Baumberg, Geometrically locked vortex lattices in semiconductor quantum fluids, Nat. Commun. 3, 1243 (2012).
[12] J. R. Ensher, D. S. Jin, M. R. Matthews, C. E. Wieman, and E. A. Cornell, Bose-Einstein Condensation in a Dilute Gas: Measurement of Energy and Ground-State Occupation, Phys. Rev. Lett. 77, 4984 (1996).

[13] T. Gao et al., Observation of non-Hermitian degeneracies in a chaotic exciton-polariton billiard, Nature (London) 526, 554 (2015).

[14] F. Baboux et al., Bosonic Condensation and DisorderInduced Localization in a Flat Band, Phys. Rev. Lett. 116, 066402 (2016).

[15] E. Wertz et al., Spontaneous formation and optical manipulation of extended polariton condensates, Nat. Phys. 6, 860 (2010).

[16] H. Deng, D. Press, S. Götzinger, G. S. Solomon, R. Hey, K. H. Ploog, and Y. Yamamoto, Quantum Degenerate Exciton-Polaritons in Thermal Equilibrium, Phys. Rev. Lett. 97, 146402 (2006).

[17] J. Kasprzak, D. D. Solnyshkov, R. André, L. S. Dang, and G. Malpuech, Formation of an Exciton Polariton Condensate: Thermodynamic versus Kinetic Regimes, Phys. Rev. Lett. 101, 146404 (2008).

[18] T. Byrnes, N. Y. Kim, and Y. Yamamoto, Exciton-polariton condensates, Nat. Phys. 10, 803 (2014).

[19] L. Dominici et al., Real-space collapse of a polariton condensate, Nat. Commun. 6, 8993 (2015).

[20] D. Sanvitto and S. Kéna-Cohen, The road towards polaritonic devices, Nat. Mater. 15, 1061 (2016).

[21] M. H. Szymańska, J. Keeling, and P. B. Littlewood, Nonequilibrium Quantum Condensation in an Incoherently Pumped Dissipative System, Phys. Rev. Lett. 96, 230602 (2006).

[22] M. Wouters and I. Carusotto, Superfluidity and Critical Velocities in Nonequilibrium Bose-Einstein Condensates, Phys. Rev. Lett. 105, 020602 (2010).

[23] J. Keeling, Superfluid Density of an Open Dissipative Condensate, Phys. Rev. Lett. 107, 080402 (2011).

[24] R. P. Smith, S. Beattie, S. Moulder, R. L. D. Campbell, and Z. Hadzibabic, Condensation Dynamics in a QuantumQuenched Bose Gas, Phys. Rev. Lett. 109, 105301 (2012).

[25] A. Janot, T. Hyart, P. R. Eastham, and B. Rosenow, Superfluid Stiffness of a Driven Dissipative Condensate with Disorder, Phys. Rev. Lett. 111, 230403 (2013).

[26] E. Altman, L. M. Sieberer, L. Chen, S. Diehl, and J. Toner, Two-Dimensional Superfluidity of Exciton Polaritons Requires Strong Anisotropy, Phys. Rev. X 5, 011017 (2015).

[27] L. V. Butov, Solid-state physics: A polariton laser, Nature (London) 447, 540 (2007).

[28] L. V. Butov and A. V. Kavokin, The behaviour of excitonpolaritons, Nat. Photonics 6, 2 (2012).

[29] B. Deveaud-Pledran, The behaviour of exciton-polaritons, Nat. Photonics 6, 205 (2012).

[30] M. Holzmann, G. Baym, J.-P. Blaizot, and F. Laloe, Superfluid transition of homogeneous and trapped twodimensional bose gases, Proc. Natl. Acad. Sci. U.S.A. 104, 1476 (2007).

[31] O. L. Berman, Y. E. Lozovik, and D. W. Snoke, Theory of Bose-Einstein condensation and superfluidity of twodimensional polaritons in an in-plane harmonic potential, Phys. Rev. B 77, 155317 (2008). 
[32] See Supplemental Material at http://link.aps.org/ supplemental/10.1103/PhysRevLett.118.016602, which includes Refs. [33-47], for a review of microcavity polaritons and previous efforts in achieving condensation in thermal equilibrium. The energy distributions $\mathrm{N}(\mathrm{E})$ at different temperatures are also included in SM.

[33] A. Kavokin, J. J. Baumberg, G. Malpuech, and F. P. Laussy, Microcavities (Oxford Science Publications, New York, 2007).

[34] H. Deng, Exciton-polariton Bose-Einstein condensation, Rev. Mod. Phys. 82, 1489 (2010).

[35] D. W. Snoke and P. Littlewood, Polariton condensates, Phys. Today 63 8, 42 (2010).

[36] I. Carusotto and C. Cuiti, Quantum fluids of light, Rev. Mod. Phys. 85, 299 (2013).

[37] J. J. Hopfield, Theory of the contribution of excitons to the complex dielectric constant of crystals, Phys. Rev. 112, 1555 (1958).

[38] S. A. Moskalenko and D.W. Snoke, Bose-Einstein Condensation of Excitons and Biexcitons (Cambridge University Press, Cambridge, England, 2000).

[39] C. Pethick and H. Smith, Bose-Einstein Condensation in Dilute Gases, 1st ed. (Cambridge University Press, Cambridge, England, 2002).

[40] L. P. Pitaevskii and S. Stringari, Bose-Einstein Condensation, International Series of Monographs on Physics (Oxford University Press, New York, 2003).

[41] H. Deng, G. Weihs, D. W. Snoke, J. Bloch, and Y. Yamamoto, Polariton lasing vs. photon lasing in a semiconductor microcavity, Proc. Natl. Acad. Sci. U.S.A. 100, 15318 (2003).

[42] M. Richard, J. Kasprzak, R. Romestain, R. André, and L. S. Dang, Spontaneous Coherent Phase Transition of Polaritons in CdTe Microcavities, Phys. Rev. Lett. 94, 187401 (2005).

[43] K. E. O'Hara and J. P. Wolfe, Relaxation kinetics of excitons in cuprous oxide, Phys. Rev. B 62, 12909 (2000).

[44] D. W. Snoke, J. P. Wolfe, and A. Mysyrowicz, Evidence for Bose-Einstein condensation of excitons in $\mathrm{Cu}_{2} \mathrm{O}$, Phys. Rev. B 41, 11171 (1990).

[45] G. Roumpos et al., Power-Law decay of the spatial correlation function in exciton-polariton condensates, Proc. Natl. Acad. Sci. U.S.A. 109, 6467 (2012).
[46] G. Dagvadorj, J. M. Fellows, S. Matyjaskiewicz, F. M. Marchetti, I. Carusotto, and M. H. Szymanska, Nonequilibrium Phase Transition in a Two-Dimensional Driven Open Quantum System, Phys. Rev. X 5, 041028 (2015).

[47] N. S. Voronova, A. A. Elistratov, and Y. E. Lozovik, Detuning-Controlled Internal Oscillations in an ExcitonPolariton Condensate, Phys. Rev. Lett. 115, 186402 (2015).

[48] M. Steger, C. Gautham, D. W. Snoke, L. Pfeiffer, and K. West, Slow reflection and two-photon generation of microcavity exciton-polaritons, Optica 2, 1 (2015).

[49] M. Steger, G. Liu, B. Nelsen, C. Gautham, D. W. Snoke, R. Balili, L. Pfeiffer, and K. West, Long-range Ballistic motion and coherent flow of long-lifetime polaritons, Phys. Rev. B 88, 235314 (2013).

[50] G. Tosi, G. Christmann, N. G. Berloff, P. Tsotsis, T. Gao, Z. Hatzopoulos, P. G. Savvidis, and J. J. Baumberg, Sculpting oscillators with light within a nonlinear quantum fluid, Nat. Phys. 8, 190 (2012).

[51] P. Cristofolini, A. Dreismann, G. Christmann, G. Franchetti, N. G. Berloff, P. Tsotsis, Z. Hatzopoulos, P. G. Savvidis, and J. J. Baumberg, Optical Superfluid Phase Transitions and Trapping of Polariton Condensates, Phys. Rev. Lett. 110, 186403 (2013).

[52] Y. Sun, Y. Yoon, M. Steger, G. Liu, L. N. Pfeiffer, K. West, D. W. Snoke, and K. A. Nelson, Polaritons are not weakly interacting: Direct measurement of the polariton-polariton interaction strength, Nat. Phys. (to be published).

[53] A. Askitopoulos, H. Ohadi, A. V. Kavokin, Z. Hatzopoulos, P. G. Savvidis, and P. G. Lagoudakis, Polariton condensation in an optically induced two-dimensional potential, Phys. Rev. B 88, 041308 (2013).

[54] A. Askitopoulos, T. C. H. Liew, H. Ohadi, Z. Hatzopoulos, P. G. Savvidis, and P. G. Lagoudakis, Robust platform for engineering pure-quantum-state transitions in polariton condensates, Phys. Rev. B 92, 035305 (2015).

[55] V. E. Hartwell and D. W. Snoke, Numerical simulations of the polariton kinetic energy distribution in gaas quantumwell microcavity structures, Phys. Rev. B 82, 075307 (2010).

[56] P. R. Eastham and P. B. Littlewood, Finite-size fluctuations and photon statistics near the polariton condensation transition in a single-mode microcavity, Phys. Rev. B 73, 085306 (2006). 